\title{
Correction to: Organizational Consulting for Strategic Change in a Public School in Colombia
}

\author{
Fernando Yzaguirre
}

\section{Correction to:}

\section{Chapter 13 in: J. M. Fritz (ed.), International Clinical}

Sociology, Clinical Sociology: Research and Practice, https://doi.org/10.1007/978-3-030-54584-0_13

The author's name was inadvertently published with an incorrect citation as "F. de Yzaguirre" in chapter 13. The incorrect citation has been corrected to "Yzaguirre, F." in the chapter now. 